\title{
A SIMULATION TOOL ASSISTING THE DESIGN OF A CLOSE RANGE PHOTOGRAMMETRY SYSTEM FOR THE SARDINIA RADIO TELESCOPE
}

\author{
F. Buffa ${ }^{a}$, A. Pinna ${ }^{\text {b,* }}$, G. Sanna ${ }^{b}$ \\ ${ }^{a}$ Osservatorio Astronomico di Cagliari, INAF - fbuffa@oa-cagliari.inaf.it \\ ${ }^{\mathrm{b}}$ Department of Civil, Environmental Engineering and Architecture, University of Cagliari, Italy - (andrea.pinna, topoca)@ unica.it
}

\author{
Commission V, WG V/5
}

KEY WORDS: Close Range Photogrammetry, Bundle Adjustment, Sardinia Radio Telescope

\begin{abstract}
:
The Sardinia Radio Telescope (SRT) is a $64 \mathrm{~m}$ diameter antenna, whose primary mirror is equipped with an active surface capable to correct its deformations by means of a thick network of actuators. Close range photogrammetry (CRP) was used to measure the self-load deformations of the SRT primary reflector from its optimal shape, which are requested to be minimized for the radio telescope to operate at full efficiency. In the attempt to achieve such performance, we conceived a near real-time CRP system which requires the cameras to be installed in fixed positions and at the same time to avoid any interference with the antenna operativeness. The design of such system is not a trivial task, and to assist our decision we therefore developed a simulation pipeline to realistically reproduce and evaluate photogrammetric surveys of large structures. The described simulation environment consists of (i) a detailed description of the SRT model, included the measurement points and the camera parameters, (ii) a tool capable of generating realistic images accordingly to the above model, and (iii) a self-calibrating bundle adjustment to evaluate the performance in terms of RMSE of the camera configurations.
\end{abstract}

\section{BACKGROUND}

The Sardinia Radio Telescope (http://www.srt.inaf .it) is a state-of-the-art radio telescope managed by the Italian National Institute for Astrophysics (INAF). The antenna is located $45 \mathrm{~km}$ far from Cagliari, the administrative capital of Sardinia (Italy).

SRT, shown in Figure 1 is a Gregorian alt-az mount radio telescope $70 \mathrm{~m}$ tall and $3000 \mathrm{t}$ weigh, with a $64 \mathrm{~m}$ diameter primary reflector. The alidade, a steel made structure $35 \mathrm{~m}$ tall, is able to support and move the primary reflector via 16 wheels acting on a $40 \mathrm{~m}$ diameter rail, around the azimuth axis. SRT exploits multiple focal positions and a wide frequency range. The receivers (the detectors) may be positioned in a few minutes on the appropriate focal position by means of robotic drivers giving a great "frequency agility" to the antenna.

The primary reflector is equipped with a deformation compensation system called "active surface", a mosaic of 1008 panels, forming 14 rings, actuated by 1116 electro-mechanical actuators. Each actuator share 4 (or 2 in some cases depending on the position) panel corners. The actuators may change the shape of the primary reflector allowing different geometric configurations: in particular when a receiver is located in the prime focus (i.e. the subreflector is not used) the shape is parabolic, but when the Gregorian setup is adopted (i.e. the subreflector is used) the antenna configuration becomes "shaped". In the shaped configuration the primary reflector may not be described in terms of a canonical surface but is given in terms of a lookup table. The shaped configuration reduces the multiple reflections between the optics and optimizes the antenna efficiency (Olmi and Grueff, 2006). It is worth noting that the active surface system may also be used to correct the surface deformations of the primary reflector due to the self-weight load and to the thermal stresses. The overall efficiency of a radio astronomical antenna is related, among other, to the shape accuracy of its reflector. Such dependence is expressed

\footnotetext{
${ }^{*}$ Corresponding author
}

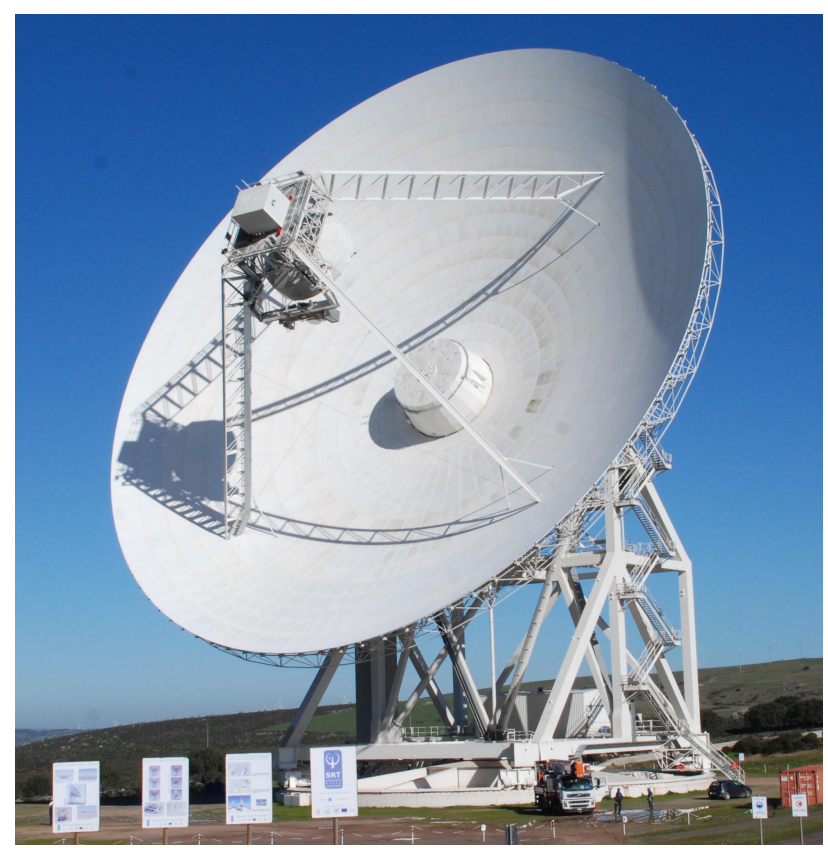

Figure 1: The Sardinia Radio Telescope.

by the so called surface-loss efficiency which is a function of the detected frequency (or equivalently of the wavelength $\lambda$ ). As a rule of thumb we can state that the RMSE to be reached is approximately equal to $\lambda / 20$. Therefore for a $100 \mathrm{GHz}$ receiver $(\lambda=3 \mathrm{~mm})$ the antenna surface must be controlled with a challenging accuracy of RMSE $\sim 150 \mu \mathrm{m}$ with respect to the ideal antenna profile. Such accuracy refers to the global alignment to be reached between the panels considered as non-deformable bodies, while the surface manufacturing accuracy of each panel in SRT case is about $70 \mu \mathrm{m}$. 
Close Range Photogrammetry (CRP) is widely used in astronomical contexts to measure the reflector deformations (Edmundson and Baker, 2001, Subrahmanyan, 2005. Shankar et al., 2009). During the SRT building phase, as well, the 1008 panels were aligned by means of CRP, reaching a global RMSE $\leq 300 \mu \mathrm{m}$, with respect to the $45^{\circ}$ elevation which may be considered as the antenna "zero" point position. The survey was performed at nighttime, using a cherry picker to hold the operator, in ideal environmental conditions (Süß et al., 2012). The panel positions were defined by means of 4032 retro-reflective targets. The panel alignment was carried out, as an iterative procedure, considering the ideal antenna profile as reference. Once the antenna profile was reached, the antenna gravitational deformation was mapped with CRP considering six different elevation positions obtaining a lookup table to be used to control each actuator. The intermediate elevation corrections are currently obtained by means of simple linear interpolation. A finite-element model has been implemented during the design studio of SRT for studying the antenna deformations; the model has been further perfected by comparison with the results of the photogrammetry surveys (Buffa et al., 2015).

As stated before this approach is suitable to take into account only gravitational deformations; this means that, in the SRT case, the antenna efficiency related to the shape is optimal till the $\mathrm{K}$ band (i.e. up to $26 \mathrm{GHz}$ ). SRT will be soon equipped with higher frequency receivers (up to $100 \mathrm{GHz}$ ), so that the thermal deformations will be relevant too and the metrological shape control will become necessarily in near real-time due to the dynamic behavior of thermal effects. Assuming that the deformation estimation will be done by CRP this would represent a challenging metrological goal if we take into account the structural complexity of such facilities and if we consider that accuracy may be strongly influenced by the environmental conditions, since the radio telescopes operate outdoors. Dealing with structures so big, metrological technique is employed to a scale which is typical of (or very close to) archaeological or architectural applications, but with requested accuracies usually achievable only in laboratory contexts. In open air the meteo-climatic conditions become a key factor for industrial CRP measurement.

In order to operate in near real-time we conceived a built-in CRP system, remotely controlled, capable of periodic measurements of the deformations of the structure. So the challenge for the metrological research group of the Sardinia Radio Telescope is to assess if a stable/robotic photogrammetric system onboard the antenna could reach the accuracy of an operator driven CRP campaign. In literature it is possible to find examples of real-time CRP applications to radio astronomical antennas (Fan et al., 2010) but in such a case we deal with (relatively) small antennas with dome enclosures that allows an optimal support for fixed or motorized cameras. In the case of large and structurally complex antennas operated in open air, as in the SRT case, the identification of optimal camera stations requires specific investigations in order to evaluate if a reliable photogrammetric triangulation network exists and if it is possible to achieve the requested accuracy degree. This may be regarded as a typical network design problem extensively studied in the technical literature which may be faced up in different ways: (i) by means of a rigorous mathematical approach in terms of zero-, first- and second-order design (Grafarend and Sansò, 1985, Fraser, 1996), (ii) by means of a "design by simulation" approach (Olague, 2000, Parian et al., 2007. Piatti and Lerma, 2013) and (iii) by means of an expert system based design (Mason and Kepuska, 1993 Mason, 1994).

In this paper we propose a "realistic" simulation system expressly designed to plan and simulate photogrammetric surveys of large infrastructures with the goal to reach millimeter and sub-millimeter accuracies. Our system mainly deals with the first-order camera positioning problem adopting a design by simulation approach. In the following sections we describe the main parts constituting the simulation pipeline, which includes:

- a complete description of the model to be studied, of the environment (in terms of obstructions blocking the line of sight), of the measurement points positions and of the camera positioning constraints;

- a tool able to generate realistic synthetic images expressed in terms of photographic image coordinates;

- a self-calibrating bundle adjustment code able to solve the mathematical problem and to evaluate the configuration under test.

In Section 2 such topics will be treated in detail, giving particular emphasis to the generation of synthetic images arising from the $3 \mathrm{D}$ coordinates of the object for a given set of interior and exterior camera parameters. Furthermore we will discuss about the choice of a rigorous bundle adjustment software and about the selection of the proper parameters for the evaluation of the results of the simulation. The simulation pipeline has been tested with different scenarios and some of them will be illustrated in Section 3 .

\section{METHODS}

Industrial applications of photogrammetry consist in measuring the position of particular points of an object with respect to a reference system. The whole procedure is typically accomplished by (i) photographing the object with an adequate number of pictures taken from well-planned positions, (ii) processing the pictures to measure the target image coordinates, (iii) entering the image coordinates to a bundle adjustment software for estimating the 3D coordinates of targets.

Through the simulation pipeline we aim at substituting the above mentioned steps (i) and (ii) by realistically calculating the image coordinates of targets for any pre-designed configuration of exterior and interior parameters of the cameras. The software would therefore allow us to evaluate the accuracy of the estimated $3 \mathrm{D}$ coordinates of targets by studying different configurations of photogrammetric cameras and stations. In the following sections we therefore describe the main parts constituting the simulation workflow, i.e. the construction of the simulation scenarios (choice of the interior and exterior parameters of cameras), the tool for computing the image coordinates of targets given a 3D model of the object and the characteristics of the cameras, the software executing the bundle adjustment process, and the measures employed for evaluating the proposed scenarios.

\subsection{The Object Environment}

In a CRP workflow, pictures of the object are processed to identify targets and to measure their image coordinates, which are then input to the bundle adjustment software for estimating the 3D position of targets. To test the performance of any possible photogrammetric scenario we therefore developed a MATLAB (The MathWorks Inc., 2013) toolbox for the computation of the image coordinates of targets. A complete knowledge of the system is needed for the software to produce the output: (i) a 3D model of the object, (ii) the targets representing the points to be measured, (iii) the ground control points, (iv) the camera exterior orientations, and (v) the camera interior orientations. 
2.1.1 Object modeling. The radio telescope includes three main parts: the basement, the alidade (which transports the steerable parts) and the antenna, which consists of the primary and the secondary mirrors (see Figure 2). Each mechanical element of

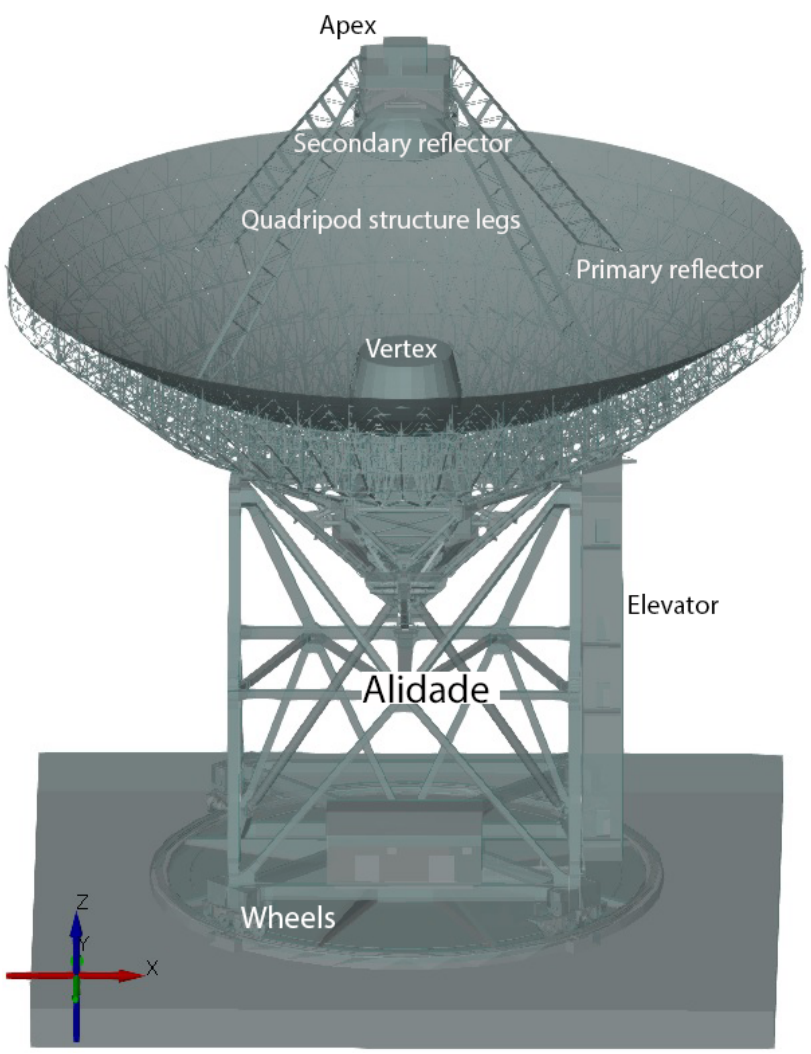

Figure 2: The Sardinia Radio Telescope 3D model.

SRT has been initially modeled with the Microstation 3D CAD software starting from the blueprint drawings and by complying with the millimetric scale tolerances of steel. The model has been later completed by incorporating direct measurements of the structure for those parts differing from design. The shaped active surface model has been built based on the theoretical profile designed to optimize the antenna efficiency. For the purposes of the simulation pipeline, we employed a simplified triangle mesh depicting the relevant parts of the case-study object: the primary mirror, the vertex, the quadripod and the apex of SRT. Coordinates are referred to a right handed reference system with the vertical $z$ axis, oriented upwards and corresponding to the azimuth axis, the $x$ axis corresponding to the elevation axis, orthogonal to $z$ and oriented towards the elevator shaft, and the $y$ axis orthogonal to $x$ and $z$. The 3D model is adequately detailed to correctly evaluate the visibility of the targets, which are arranged at the corners of the panels of the primary mirror (i.e. in the vicinity of the actuators) and approximately in their center. The triangle mesh is therefore mainly used to evaluate which targets are concealed to which cameras. It also allows to precisely define the constraints of the camera positions with respect to the reference system.

2.1.2 Target points. A set of $n_{t}=5198$ targets is used to represent the points to be measured. Each target $t$ has a circular shape and is characterized by the spatial coordinates $x^{t}, y^{t}$ and $z^{t}$ of its barycenter, the diameter $d_{t}$, and the normal versor $\mathbf{v}_{t}$ with respect to the surface of the primary mirror. Additional targets might be placed on the surface of the secondary mirror to evaluate the alignment between the two mirrors. A subset of target coordinates can be chosen as known ground control points (GCPs) in order to fix the reference frame.

2.1.3 Exterior orientations. The design of the measurement network necessitates the resolution of tightly interrelated logistical and photogrammetric issues. The logistical problem consists in the identification of the parts of SRT able to accommodate cameras that do not interfere with the motion of the antenna: few parts of the radio telescope satisfy this condition, such that any feasible configuration of cameras would yield lesser photogrammetric measurements than the theoretically optimal configuration, because it must comply with the logistical boundaries. We therefore prepared eight illustrative fundamental sets of exterior orientations (see Figure 3 with cameras positioned, accordingly to the above mentioned logistical constraints, around the edge of the primary mirror (sets $E_{1}$ to $E_{4}$ ), protruding from the apex $\left(A_{1}\right.$ and $\left.\mathrm{A}_{2}\right)$ and on the vertex roof $\left(\mathrm{V}_{1}\right.$ and $\left.\mathrm{V}_{2}\right)$. The proposed scenarios are built by selecting a number of cameras from the above sets. Additional sets of cameras, e.g. positioned on the legs of the quadripod, can be easily designed and consequently their performance evaluated. Each camera is described by the coordinates $x^{c}, y^{c}$ and $z^{c}$ of its principal point and by the angles $\omega^{c}, \phi^{c}$ and $\kappa^{c}$ (or the rotation matrix $\mathbf{R}^{c}$ ) describing the orientation of the optical axis with respect to the reference system.

2.1.4 Interior orientations. Simulated pictures can be shoot with a varied range of realistic cameras, each characterized by its own interior parameters. In particular, the synthetic cameras are defined by their the focal length $F$, the coordinates $X_{\mathrm{h}}$ and $Y_{\mathrm{h}}$ of the principal point, the parameters $A_{1}, A_{2}$ and $A_{3}$ modeling the radial distortion (Brown, 1971), $B_{1}$ and $B_{2}$ modeling the tangential distortion (Brown, 1966), $C_{1}$ and $C_{2}$ modeling the affinity and shear distortions, the width $X_{\mathrm{ss}}$ and the height $Y_{\mathrm{ss}}$ of the sensor, and its resolution in pixel with $X_{\mathrm{px}}$ and $Y_{\mathrm{px}}$. All the above parameters can be regulated to maximize the performance and the realism of the network design.

\subsection{Simulation of Target Image Coordinates}

Once the simulation scenario has been defined, the MATLAB toolbox computes for all the cameras $c$ the image coordinates $X_{t c}$ and $Y_{t c}$ of targets $t$ by solving the collinearity equations and by modeling realistic distortion elements as the radial symmetrical component $\Delta^{\mathrm{r}}$, the radial asymmetrical and tangential components $\Delta^{\mathrm{t}}$ and the affine component $\Delta^{\mathrm{a}}$ (Luhmann et al., 2006). The image coordinates $X_{t c}^{\mathrm{d}}$ and $Y_{t c}^{\mathrm{d}}$ of the target defined within the distortion model are therefore:

$$
\left\{\begin{array}{l}
X_{t c}^{\mathrm{d}}=X_{t c}+\Delta_{X_{t c}}^{\mathrm{r}}+\Delta_{X_{t c}}^{\mathrm{t}}+\Delta_{X_{t c}}^{\mathrm{a}} \\
Y_{t c}^{\mathrm{d}}=Y_{t c}+\Delta_{Y_{t c}}^{\mathrm{r}}+\Delta_{Y_{t c}}^{\mathrm{t}}+\Delta_{Y_{t c}}^{\mathrm{a}}
\end{array}\right.
$$

Furthermore, only a fraction of the above targets satisfies the visibility and accuracy constraints for being assigned to the image corresponding to the exterior orientation $c$. In particular, a target $t$ with coordinates $\left(X_{t c}^{\mathrm{d}}, Y_{t c}^{\mathrm{d}}\right)$ is represented in image $c$ if the following five conditions are satisfied.

Field of view. The image coordinates do not exceed the sensor boundaries: $\left|X_{t c}^{\mathrm{d}}\right|<X_{\mathrm{ss}} / 2$ and $\left|Y_{t c}^{\mathrm{d}}\right|<Y_{\mathrm{ss}} / 2$.

Visibility. The target is completely visible from the camera, i.e. is not obscured by any part of SRT (e.g. the quadripod legs).

Direction. The target is placed in front of the camera $c$ (and not behind): $\left[\left(x^{t}-x^{c}\right),\left(y^{t}-y^{c}\right),\left(z^{t}-z^{c}\right)\right] \cdot \mathbf{v}_{c}<0$, where $\mathbf{v}_{c}$ is the optical axis of camera $c$. 
Edge (E1)

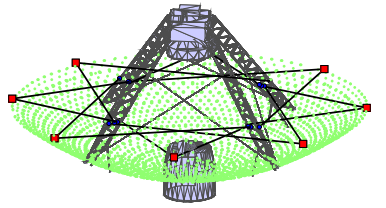

Edge (E3)

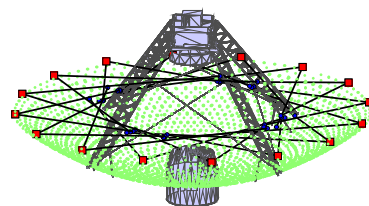

Apex (A1)

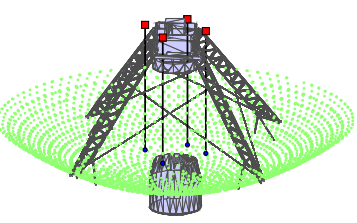

Vertex (V1)

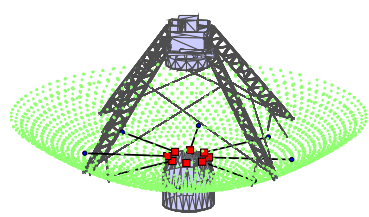

Edge (E2)

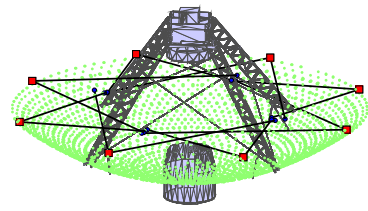

Edge (E4)

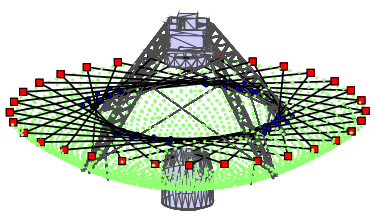

Apex (A2)

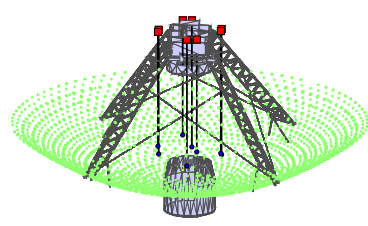

Vertex (V2)

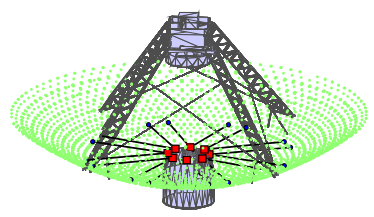

Figure 3: Fundamental sets of cameras: the red squares correspond to the cameras, and the black lines to the optical axes; targets are represented by the green dots.

Distance. The target is close enough to the camera so that its diameter is represented with at least $p^{\star}=10$ pixels (Luhmann, 2011), i.e. the target is large enough for an adequately accurate measurement of the coordinates of its barycenter.

Incidence angle. The angle $\alpha_{t c}$ between the optical axis $\mathbf{v}_{c}$ and the normal to the target $\mathbf{n}_{t}$ is smaller than a threshold angle $\alpha^{\star}$.

Requirements about distance and incidence angle are here deliberately modeled in a simple way. In fact, in a related project regarding SRT, a more realistic simulator capable of generating the images, inclusive of targets, has been developed (Saba, 2015). Such software will be employed for the evaluation of the most interesting configurations preselected by the here presented simulator of target image coordinates.

Finally, once the set of image targets satisfying the above requirements has been selected, a normally distributed noise with a given $\sigma_{\mathrm{n}}$ is added to the already computed image coordinates.

The simulation software is furthermore capable of providing statistics about targets and images, e.g. the number of images depicting a particular target or the number of visible targets for each image. Each image can be therefore classified accordingly to the average distance of targets, the average incidence angles, the ratio between the convex surface containing all the targets and the whole area of the image, etc. The simulation toolbox provides tools for changing the width/height ratio of the sensor, enhancing the pixel resolution, adapting the focal length, etc.

\subsection{Bundle Adjustment}

The image coordinates of targets, a set of GCPs, and the initial values for the camera interior parameters (e.g. focal length) are input to AICON 3D Studio (http://aicon3d.com) and MicMac (http://logiciels.ign.fr/?Micmac), both software capable of running a self-calibrating bundle adjustment.

AICON 3D Studio (AICON 3D Systems, 2015) is a thoroughly tested software for applications of industrial photogrammetry, and is also being adopted for the on-site measurement of SRT. Besides its reliability, this commercial software also quantifies the quality of the photogrammetric measurements by providing the variancecovariance matrix. Nonetheless, the usage of AICON 3D Studio requests a constant interaction with the user, and therefore is unsuitable for the batch processing of simulation scenarios.

MicMac (Pierrot-Deseilligny, 2014) is an open source software performing a complete self-calibrating bundle adjustment for the generation of 3D point clouds in the fields of architecture, archeology and cartography. The name MicMac stands for a broader suite of tools developed for transforming an object, photographed from several viewpoints, into a 3D model. In our study we used the tool Apero (Pierrot-Deseilligny and Clery, 2011), where the pin hole camera model is rigorously implemented, to estimate interior and exterior orientations based on the tie-point measurements, i.e. the input target image coordinates. We therefore run Apero via the simplified tools Tapas (for the computation of relative orientations), GCPBascule (for transforming the relative orientations into a reference system described by the GCPs) and Campari (for the compensation of the image coordinates and the GCPs), to fit a 12-parameter model (Fraser, 1997). Finally, the $3 \mathrm{D}$ coordinates of targets are obtained by running AperiCloud with the interior and exterior orientations produced by Campari as input. The above are command line tools and can be integrated in the simulation pipeline for the batch evaluation of different scenarios. We therefore can use MicMac for running the bundle adjustment process for testing and evaluating hundreds configurations of cameras, while AICON 3D Studio can be employed to produce benchmark results on a small subset of simulation scenarios for comparison. Oppositely to AICON 3D Studio, MicMac does not compute a variance-covariance matrix to measure the quality of the bundle adjustment.

An essential component of the simulation pipeline is dedicated to (i) formatting the input data as requested by MicMac and AICON 3D Studio software and (ii) converting the output data produced by the bundle adjustment tools in the same format as the input data, to immediately compare "true" and measured values.

\subsection{Evaluation of performances}

Both AICON 3D Studio and MicMac yield, respectively, the measured spatial coordinates $\left(x_{\mathrm{A}}^{t}, y_{\mathrm{A}}^{t}, z_{\mathrm{A}}^{t}\right)$ and $\left(x_{\mathrm{M}}^{t}, y_{\mathrm{M}}^{t}, z_{\mathrm{M}}^{t}\right)$ of the $n_{t}$ targets, which can be compared with the "true" values $\left(x^{t}, y^{t}\right.$, $z^{t}$ ). Equally, the estimates of interior and exterior parameters can be compared with the corresponding input values too. A summarizing measure of the goodness of a simulation scenario is the 
RMSE between the input and the measured positions of targets:

$$
\operatorname{RMSE}=\sqrt{\frac{\sum_{t=1}^{n_{t}}\left[\left(x_{\mathrm{A}}^{t}-x^{t}\right)^{2}+\left(y_{\mathrm{A}}^{t}-y^{t}\right)^{2}+\left(z_{\mathrm{A}}^{t}-z^{t}\right)^{2}\right]}{n_{t}}}
$$

The RMSE is also computed on subsets of targets, selected by their radial distance from the $z$ axis of the reference system: this technique allows for evaluating the precision of the measurement on different panel rings, since e.g. a more accurate alignment is desired for the inner panels than the exterior ones.

The error on the measured position of target $t$ can be conveyed by computing the distance between the estimated target and the "true" target. As an example, the error on the estimate of target $t$ by AICON 3D Studio is expressed by:

$$
\mathrm{E}_{t}=\sqrt{\left(x_{\mathrm{A}}^{t}-x^{t}\right)^{2}+\left(y_{\mathrm{A}}^{t}-y^{t}\right)^{2}+\left(z_{\mathrm{A}}^{t}-z^{t}\right)^{2}}
$$

The error can be measured for each coordinate $x^{t}, y^{t}$ and $z^{t}$ of target $t$, separately:

$$
\mathrm{E}_{t}^{x}=x_{\mathrm{A}}^{t}-x^{t}, \quad \mathrm{E}_{t}^{y}=y_{\mathrm{A}}^{t}-y^{t}, \quad \mathrm{E}_{t}^{z}=z_{\mathrm{A}}^{t}-z^{t}
$$

The magnitude of the above errors on target estimates can be also graphically shown by plotting the targets with colored dots after mapping the corresponding error values into a colormap. Such plots are particularly useful for detecting bias in the measurements or for revealing which parts, if any, of the object are measured with e.g. lesser or higher precision with respect to the average value, and therefore might quickly point to a correction of the camera configuration.

\section{SIMULATION SCENARIOS}

In this section we show the analysis performed on two case tests carried out in order to check the simulation workflow. The first case, "Scenario A", was a real photogrammetry survey (Süß et al., 2012), kindly provided by Heiko Paluszek (Sigma3D). The scenario, entirely reconstructed by the simulator, is composed by 218 synthetic images taken from the stations shown in Figure 4. The distribution of the number of homologous image rays concurring to the assessment of target coordinates, i.e. the number of times a target is measured in different images (see Figure 8 , top) shows the downsides of real photogrammetry surveys for such a large structure: the operators cannot take pictures homogeneously, and their range of movement is restricted by the length of the crane and by the structural constraints of the model to be studied. In the original survey 10 control points were used to materialize the reference frame, which were replicated in this simulation study. The second case, "Scenario B", was built based on the fundamental sets of cameras shown in Figure 3 In Scenario B we generated 164 synthetic images taken from apex, primary reflector edge and vertex, leading to the more uniform distribution of homologous ray occurrences (Figure 9 top). In order to investigate the performance of both bundle adjustment software according to the positioning and to the number of cameras, we also evaluated subsets of Scenario B. To simulate all scenarios we used the same interior camera parameters and fixed the same control points of Scenario A. We also set the standard deviation of the noise applied on image coordinates to $\sigma_{\mathrm{n}}=5 \mu \mathrm{m}$.

\subsection{Results of Bundle Adjustment in Scenario A}

The self-calibrating bundle adjustment by AICON 3D Studio provided a global $\mathrm{RMSE}_{\mathrm{A}}=0.236 \mathrm{~mm}$, while that by MicMac produced a larger value: RMSE$_{M}=0.293 \mathrm{~mm}$. The better performance of AICON 3D Studio is confirmed locally for each ring

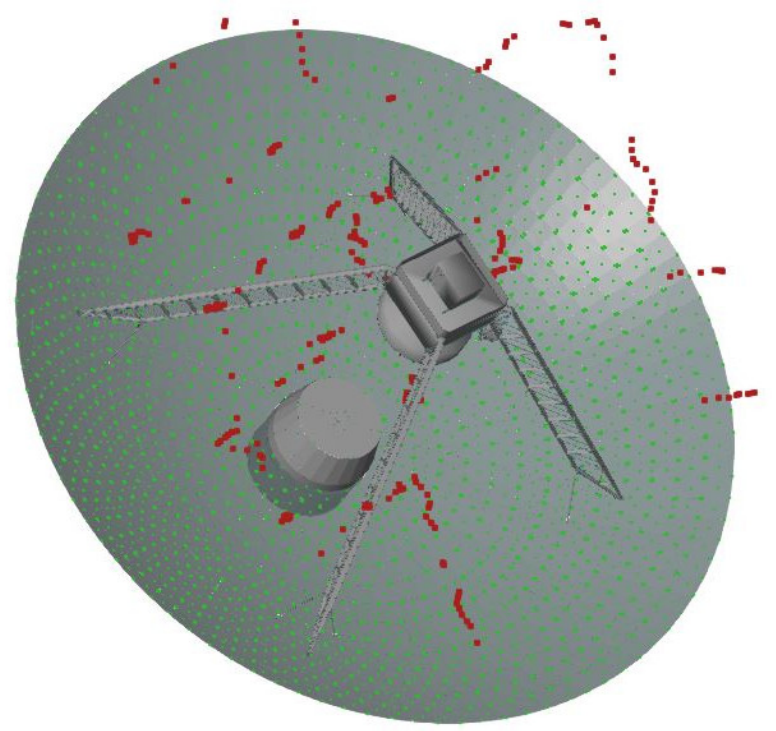

Figure 4: Positions of cameras (red dots) for Scenario A. Targets are represented on the surface of the primary mirror as green dots.

of targets, as shown in Figure 5 the error is constantly lower in AICON 3D Studio with respect to MicMac. Moreover, in both cases the locally evaluated RMSE by rings increases progressively along the radial distance. This is particularly relevant for our study because the most sensitive parts of the primary mirror with respect to the antenna efficiency are those closer to the vertex. Again, this tendency is confirmed by the spatial distribu-

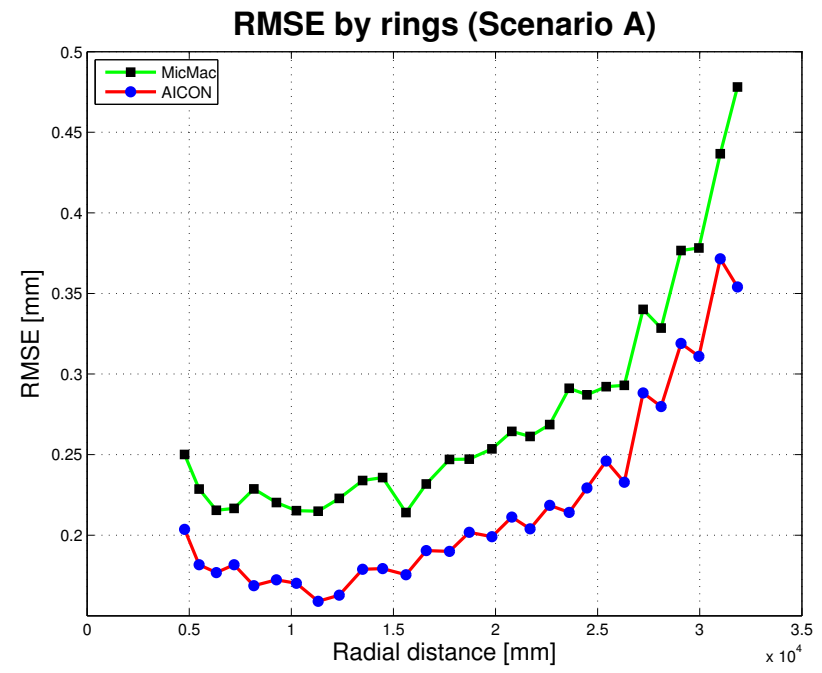

Figure 5: RMSE computed for each ring of targets, identified by their radial distance, for Scenario A.

tions of error (Figure 8 middle for AICON 3D Studio, bottom for MicMac), which mirror the RMSE performances. In fact, the area containing "blue" targets (points estimated with higher precision) is more extended for AICON 3D Studio than MicMac, where the estimate of the target positions seems to be affected by the non homogeneous distribution of homologous ray occurrences.

In Table 1 we compare the estimated values of interior camera parameters with respect to the "true" values used to simulate the image target coordinates. For both bundle adjustment software, the parameter estimates are close to the "true" values, proving the 
correct implementation of the simulator; it is worth noting that the focal length $F$ is underestimated by both AICON 3D Studio and MicMac.

\begin{tabular}{|c|c|c|c|}
\hline$[\mathrm{mm}]$ & Input & AICON & MicMac \\
\hline$F$ & 28.661 & 28.330 & 28.434 \\
$A_{1}$ & $-9.070 \mathrm{E}-5$ & $-9.390 \mathrm{E}-5$ & $-9.282 \mathrm{E}-5$ \\
$A_{2}$ & $1.570 \mathrm{E}-7$ & $1.663 \mathrm{E}-7$ & $1.644 \mathrm{E}-7$ \\
$A_{3}$ & $0.000 \mathrm{E}+0$ & $1.125 \mathrm{E}-13$ & $-5.083 \mathrm{E}-27$ \\
$B_{1}$ & $-4.350 \mathrm{E}-6$ & $-4.448 \mathrm{E}-6$ & $-4.384 \mathrm{E}-6$ \\
$B_{2}$ & $8.770 \mathrm{E}-6$ & $8.929 \mathrm{E}-6$ & $8.844 \mathrm{E}-6$ \\
$C_{1}$ & $-3.540 \mathrm{E}-5$ & $-3.539 \mathrm{E}-5$ & $-3.544 \mathrm{E}-5$ \\
$C_{2}$ & $6.470 \mathrm{E}-5$ & $6.440 \mathrm{E}-5$ & $6.458 \mathrm{E}-5$ \\
\hline
\end{tabular}

Table 1: Comparison of the "true" and estimated interior camera parameters for Scenario A.

\subsection{Results of Bundle Adjustment in Scenario B}

In this section we deal with the design of a CRP system where the cameras are physically bound to the radio telescope. The ultimate goal of the project is, in fact, the setup of a permanent infrastructure to measure in near real-time the deformation of the primary reflector of SRT. We therefore studied the performance of MicMac and AICON 3D Studio in computing the spatial coordinates of targets by processing the measurements produced by different camera configurations. In particular, we built 10 scenarios $\left(\mathrm{B}_{1^{-}}\right.$ $\mathrm{B}_{10}$ ) by joining the fundamental sets of cameras (Figure 3) as illustrated in Table 2 As expected, the global RMSE improves with the availability of additional images (Figure 6), with $\mathrm{B}=\mathrm{B}_{10}$ being the most successful configuration of cameras. On the other hand, almost the same result is obtained by Scenario $B_{7}$, which is better than $B_{8}$ and $B_{9}$, i.e. the extra images taken from the vertex (set $\mathrm{V}_{2}$ ) seem to deteriorate the performance. This is not confirmed by scenarios $B_{5}$ and $B_{6}$, where the images from cameras of set $\mathrm{V}_{2}$ yield an improvement in the RMSE.

\begin{tabular}{|c|c|c|c|c|c|c|c|c|c|}
\hline & \#I & $\mathrm{E}_{1}$ & $\mathrm{E}_{2}$ & $\mathrm{E}_{3}$ & $\mathrm{E}_{4}$ & $\mathrm{~A}_{1}$ & $\mathrm{~A}_{2}$ & $\mathrm{~V}_{1}$ & $\mathrm{~V}_{2}$ \\
\hline $\mathrm{B}_{1}$ & 28 & $\checkmark$ & & & & $\checkmark$ & & $\checkmark$ & \\
$\mathrm{B}_{2}$ & 36 & $\checkmark$ & & & & $\checkmark$ & & & $\checkmark$ \\
$\mathrm{B}_{3}$ & 44 & $\checkmark$ & $\checkmark$ & & & $\checkmark$ & & $\checkmark$ & \\
$\mathrm{B}_{4}$ & 52 & $\checkmark$ & $\checkmark$ & & & $\checkmark$ & & & $\checkmark$ \\
$\mathrm{B}_{5}$ & 76 & $\checkmark$ & $\checkmark$ & $\checkmark$ & & $\checkmark$ & & $\checkmark$ & \\
$\mathrm{B}_{6}$ & 84 & $\checkmark$ & $\checkmark$ & $\checkmark$ & & $\checkmark$ & & & $\checkmark$ \\
$\mathrm{B}_{7}$ & 140 & $\checkmark$ & $\checkmark$ & $\checkmark$ & $\checkmark$ & $\checkmark$ & & $\checkmark$ & \\
$\mathrm{B}_{8}$ & 148 & $\checkmark$ & $\checkmark$ & $\checkmark$ & $\checkmark$ & $\checkmark$ & & & $\checkmark$ \\
$\mathrm{B}_{9}$ & 156 & $\checkmark$ & $\checkmark$ & $\checkmark$ & $\checkmark$ & $\checkmark$ & & $\checkmark$ & $\checkmark$ \\
$\mathrm{B}_{10}$ & 164 & $\checkmark$ & $\checkmark$ & $\checkmark$ & $\checkmark$ & $\checkmark$ & $\checkmark$ & $\checkmark$ & $\checkmark$ \\
\hline
\end{tabular}

Table 2: Number of images and fundamental sets of cameras composing the subsets of Scenario B.

The bundle adjustment analysis performed on the 164 synthetic images of Scenario B reveals that MicMac apparently outperforms AICON 3D Studio by registering a $\mathrm{RMSE}_{\mathrm{M}}=0.279 \mathrm{~mm}$, while the commercial software yields a $\mathrm{RMSE}_{\mathrm{A}}=0.339 \mathrm{~mm}$. A closer look at Figure 7 shows that the error is differently distributed along the radial distance: AICON 3D Studio estimates more precisely the targets placed in the first $15 \mathrm{~m}$ of the primary mirror, while MicMac performs better in measuring the targets radially distant more than $15 \mathrm{~m}$. The different bundle adjustment algorithms implemented in AICON 3D Studio and MicMac show their diverse behavior within the spatial distribution of errors (Figure 9 middle for AICON 3D Studio, bottom for Mic$\mathrm{Mac})$. While in MicMac target estimates the error is roughly inversely correlated to the number of homologous ray occurrences (Figure 9 top), AICON 3D Studio optimizes the performance in the inner rings, closer to the vertex, to the detriment of the accuracy on the outer targets, as already shown in Figure 7 Estimates

\section{Global RMSE after BA by MicMac and AICON}

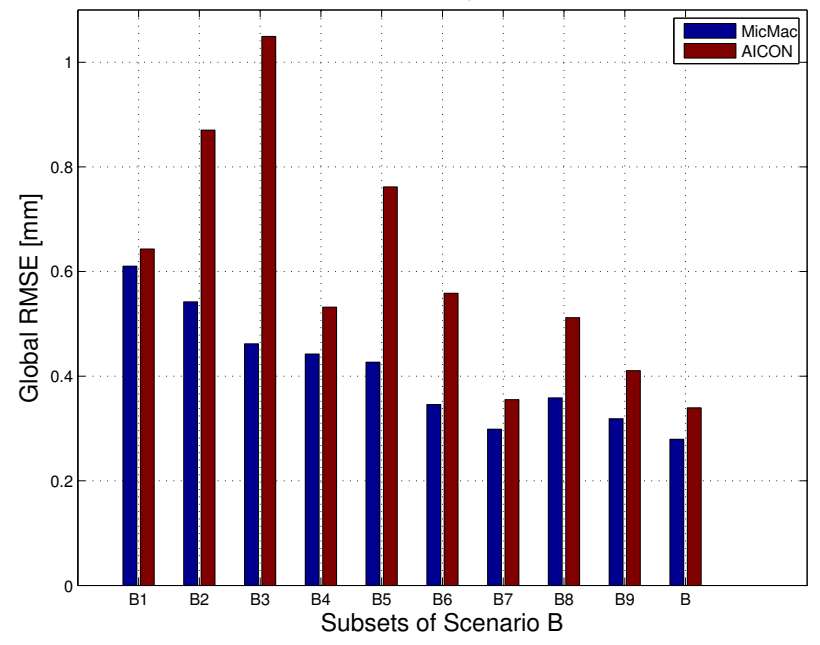

Figure 6: RMSE measured for subsets of Scenario B (described in Table 2, sorted by increasing number of images.

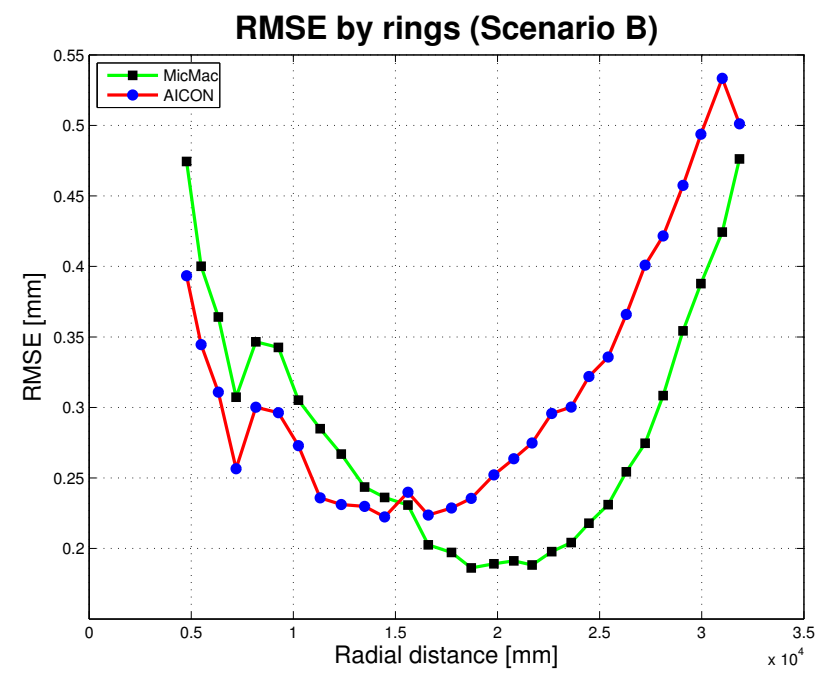

Figure 7: RMSE computed for each ring of targets, identified by their radial distance, for Scenario B.

of the interior camera parameters for Scenario B are shown in Table 3. where MicMac seems to be slightly more accurate than AICON 3D Studio, even if both software are capable of getting close to the "true" values and hence demonstrating the correct behavior of the self-calibrating bundle adjustments.

The computational time for MicMac tools to complete the bundle adjustment run was of about $40 \mathrm{~min}$ (Scenario A) and $75 \mathrm{~min}$ (Scenario B), while AICON 3D Studio completed the runs in just $5 \mathrm{~min}$ and $10 \mathrm{~min}$, respectively. Such disparity in the computational times might explain, in addition to differently implemented strategies in the bundle adjustment algorithms, the slightly lower performance of AICON 3D Studio in Scenario B configurations. Moreover, the software are executed on different machines: MicMac on a single $3.3 \mathrm{GHz}$ processor, while AICON 3D Studio on a single $2.8 \mathrm{GHz}$ processor. However, while AICON 3D Studio requires the user guidance, MicMac works entirely in batch mode and therefore the whole CRP process could be automated. 

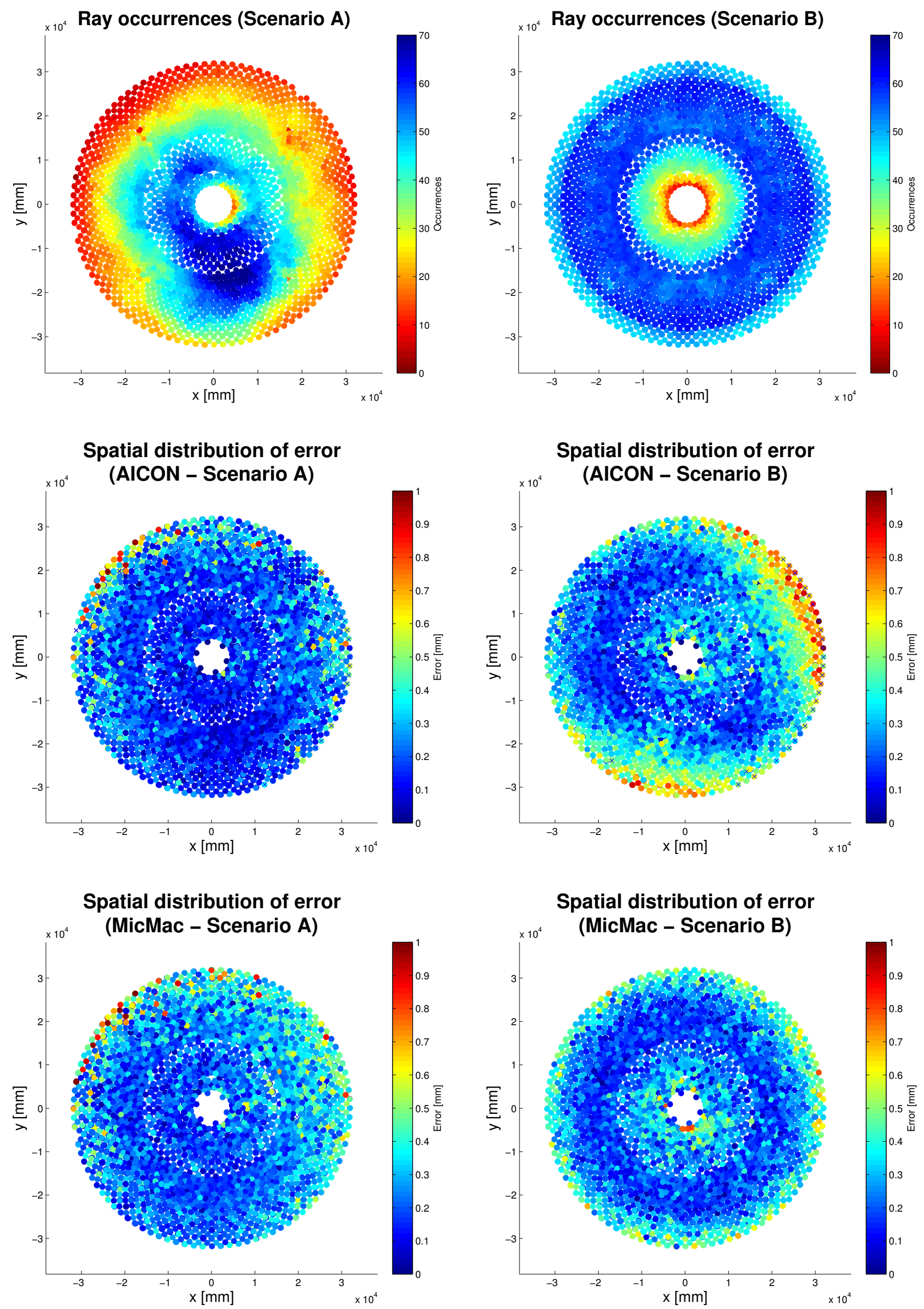

Figure 8: Distribution of homologous ray occurrences (top) and spatial distribution of error (middle and bottom) for Scenario A.

Figure 9: Distribution of homologous ray occurrences (top) and spatial distribution of error (middle and bottom) for Scenario B. 


\begin{tabular}{|c|c|c|c|}
\hline$[\mathrm{mm}]$ & Input & AICON & MicMac \\
\hline$F$ & 28.661 & 28.330 & 28.433 \\
$A_{1}$ & $-9.070 \mathrm{E}-5$ & $-9.391 \mathrm{E}-5$ & $-9.285 \mathrm{E}-5$ \\
$A_{2}$ & $1.570 \mathrm{E}-7$ & $1.663 \mathrm{E}-7$ & $1.646 \mathrm{E}-7$ \\
$A_{3}$ & $0.000 \mathrm{E}+0$ & $5.315 \mathrm{E}-14$ & $-5.696 \mathrm{E}-27$ \\
$B_{1}$ & $-4.350 \mathrm{E}-6$ & $-4.555 \mathrm{E}-6$ & $-4.445 \mathrm{E}-6$ \\
$B_{2}$ & $8.770 \mathrm{E}-6$ & $8.970 \mathrm{E}-6$ & $8.945 \mathrm{E}-6$ \\
$C_{1}$ & $-3.540 \mathrm{E}-5$ & $-2.307 \mathrm{E}-5$ & $-3.323 \mathrm{E}-5$ \\
$C_{2}$ & $6.470 \mathrm{E}-5$ & $6.879 \mathrm{E}-5$ & $6.553 \mathrm{E}-5$ \\
\hline
\end{tabular}

Table 3: Comparison of the "true" and estimated interior camera parameters for Scenario B.

\section{CONCLUSIONS}

We presented a simulation pipeline for evaluating the performance of logistically bound camera configurations in measuring with sub-millimetric accuracy the panel deformations of the Sardinia Radio Telescope by means of close range photogrammetry. The main part of the simulation workflow is a MATLAB toolbox capable of computing the image coordinates of targets given an input scenario consisting of the 3D model of the object under study, the positions of the targets, the interior and exterior camera parameters, and a set of ground control points. We solved the selfcalibrating bundle adjustment for a pair of exemplifying scenarios, and evaluated the results by especially comparing AICON 3D Studio and MicMac. The two software, while delivering quantitatively similar performances in terms of RMSE and estimate of interior camera parameters, measured with different accuracy the target positions depending on their radial distance from the reflector vertex. Investigating the reasons behind such different behaviors is beyond the scope of our study; moreover, while MicMac authors describe their work (Pierrot-Deseilligny and Clery, 2011), AICON 3D Studio is a commercial software whose bundle adjustment algorithm is not available for inspection.

The unacceptably long computational time makes MicMac unsuitable for the near real-time monitoring of SRT panel deformations. Nonetheless, thanks to its capability of running in batch mode, MicMac is still valuable for automatically processing the simulated target image measurements from a potentially unlimited collection of camera configurations, which can be therefore evaluated. In particular, the interior and exterior camera parameters can be optimized to reach a tradeoff between measurement accuracy and logistical constraints. A selection of the most promising scenarios can be then validated by AICON 3D Studio, and later concretely implemented and tested within SRT.

\section{REFERENCES}

AICON 3D Systems, 2015. AICON 3D Studio user manual.

Brown, D. C., 1966. Decentering distortion of lenses. Photogrammetric Engineering 32(3), pp. 444-462.

Brown, D. C., 1971. Close range camera calibration. Photogrammetric Engineering 37, pp. 855-866.

Buffa, F., Causin, A., Cazzani, A., Poppi, S., Sanna, G., Solci, M., Stochino, F. and Turco, E., 2015. The Sardinia Radio Telescope: a comparison between close range photogrammetry and FE models [published online before print on December 23, 2015]. Mathematics and Mechanics of Solids.

Edmundson, K. and Baker, L., 2001. Photogrammetric measurement of the Arecibo primary reflector surface. In: 17th annual Coordinate Measurement Systems Committee Conference.
Fan, Q., Fang, S., Zuo, Y., Li, Y., Sun, J., Yang, J., Li, J., $\mathrm{Xu}, \mathrm{Y}$. and He, D., 2010. Application of digital industrial photogrammetric technology to measure the surface accuracy of 13.7 $\mathrm{m}$ millimeter-wave radio telescope antenna. Acta Astronomica Sinica 51, pp. 210-216.

Fraser, C. S., 1996. Close range photogrammetry and machine vision. Whittles Publishing, chapter Network design, pp. 256281.

Fraser, C. S., 1997. Digital camera self-calibration. ISPRS Journal of Photogrammetry and Remote Sensing 52(4), pp. 149-159.

Grafarend, E. W. and Sansò, F., 1985. Optimization and design of geodetic networks. Springer-Verlag.

Luhmann, T., 2011. 3D imaging: how to achieve highest accuracy. In: SPIE Optical Metrology, International Society for Optics and Photonics, pp. 808502-808502.

Luhmann, T., Robson, S., Kyle, S. and Harley, I., 2006. Close range photogrammetry: principles, methods and applications. Whittles Publishing.

Mason, S. O., 1994. Expert system-based design of photogrammetric networks. PhD thesis, Institute of Geodesy and Photogrammetry, ETH Zurich, Switzerland.

Mason, S. O. and Kepuska, V., 1993. On the representation of close range network design knowledge. International Archives of Photogrammetry and Remote Sensing 29, pp. 446-446.

Olague, G., 2000. Design and simulation of photogrammetric networks using genetic algorithms. In: Proceedings of the 2000 Meeting of the Americal Society for Photogrammetry and Remote Sensing.

Olmi, L. and Grueff, G., 2006. SRT: design and technical specifications. Memorie della Società Astronomica Italiana - Supplementi 10, pp. 19.

Parian, J. A., Gruen, A. and Cozzani, A., 2007. Monitoring of the reflectors of ESA's Planck telescope by close-range photogrammetry. Journal of Applied Geodesy 1(3), pp. 137-145.

Piatti, E. J. and Lerma, J. L., 2013. Virtual worlds for photogrammetric image-based simulation and learning. The Photogrammetric Record 28(141), pp. 27-42.

Pierrot-Deseilligny, M., 2014. MicMac, Apero, Pastis and other beverages in a nutshell! Institut Géographique National.

Pierrot-Deseilligny, M. and Clery, I., 2011. Apero, an open source bundle adjustment software for automatic calibration and orientation of set of images. International Archives of the Photogrammetry, Remote Sensing and Spatial Information Sciences 38, pp. 269-276.

Saba, A., 2015. SRT Photogrammetry Simulator [available online at http://goo.gl/PhQPed]. Technical report, Osservatorio Astronomico di Cagliari (INAF).

Shankar, N. U., Duraichelvan, R., Ateequlla, C., Nayak, A., Krishnan, A., Yogi, M., Rao, C. K., Vidyasagar, K., Jain, R., Mathur, P., Govinda, K. V., Rajeev, R. B. and Danabalan, T. L., 2009. Photogrammetric measurements of a 12-metre preloaded parabolic dish antenna. In: Proceedings of the National Workshop on the Design of Antenna and Radar Systems.

Subrahmanyan, R., 2005. Photogrammetric measurement of the gravity deformation in a cassegrain antenna. IEEE Transactions on Antennas and Propagation 53(8), pp. 2590-2596.

Süß, M., Koch, D. and Paluszek, H., 2012. The Sardinia Radio Telescope optical alignment. In: Proceedings SPIE, Vol. 8444.

The MathWorks Inc., 2013. MATLAB R2013a (version 8.1.0). 